\title{
Pyloric stenosis of infancy and newborn prescription of ppi drugs-the acid test
}

\begin{abstract}
There is mounting evidence that the inheritance of Primary Hyperacidity coupled with the normal developmental increase of acid production at around 3weeks of age, produces sufficient peak hyperacidity to trigger repeated contractions of the pyloric sphincter and work hypertrophy. Since this peak acidity is temporary and naturally resolves, any stimulus which reduces acidity even temporarily during these critical 3-4weeks would theoretically lead to a long term cure and reduce the incidence of PS. During the last 3 decades PPI drugs have been increasingly used in modern pediatric practice in the Western world.In new-borns less than 1/12 of age PPI prescriptions have more than doubled between 2004 and 2008. usually for an alleged diagnosis of GERD. During the last 2 decades there has been a significant,unusual and uniform decline in the incidence of PS in the western world. The demographics of the decline give no support to the proposal that the back to sleep campaign for Sudden Infant Death Syndrome (SIDS) is the explanation. It is here asserted that the falling incidence of PS is caused by PPI induced loss of acidity at a critical time in the evolution of the disease process.
\end{abstract}

Volume 6 Issue 4 - 2017

Rogers IM
Formerly Prof Surgery, AIMST University, UK

Correspondence: Rogers IM, Formerly Prof. Surgery, AIMST University, Malaysia, FRCS, FRCP, PGCM ed Edu, 46 Whitburn Road, Cleadon, Sunderland SR67QS, UK,

Email irogers2000@hotmail.com

Received: March 09, 2017| Published: April 04, 2017

Keywords: pyloric stenosis of infancy, primary hyperacidity, etiology, decline in incidence of PS, GERD in infants, PPI drugs

Abbreviations: PS, pyloric stenosis of infancy; GERD, gastroesophageal reflux in infancy; SIDS, sudden infant death syndrome

\section{Introduction}

Much ink has been spilled on the subject of PS since the first description more than 100years ago. The curious clinical features have been refined and documented endlessly for little obvious advantage.In more recent years theories of cause have been given more attention. Extraordinarily none of these theories seeks to explain the clinical features. The peculiar time sensitive features and the tendency to long term cure after a certain survivable age have been particularly and spectacularly ignored. It is little wonder that these theories have come to nothing. The hyperacidity theory ${ }^{1}$ explains all the known clinical features and is based on the knowledge that PS babies secrete more acid than normal even when gastric outlet has been relieved. Indeed a canine model exists which will produce PS in new-born puppy dogs when their gastric acid secretion is artificially raised by pentagastrin injections given to their mothers before birth. ${ }^{2}$ The rapid rise of fasting gastrin from Day 1to Day 4 in normal human development is associated with no post-feed rise and reaches levels beyond the adult range. This elevated fasting gastrin only falls at around 2 months of age when a post-prandial increase is detected ${ }^{3,4}$ (Figure 1). Such observations suggest two things.

1. The gastrin secretion is initially maximally secreted. The small difference between maximal stimulated acid secretion and basal secretion during these early weeks is consistent with this interpretation. $^{5}$

2. At 3 weeks the normal feedback between gastrin and acidity matures. The peak acidity induced by unrestrained gastrin secretion thereafter reduces as it comes under feed-back control at around 3 weeks of age. This theory is well substantiated by the graph of acid parameters in normal babies measured by Agunod (Figure 2). ${ }^{6}$

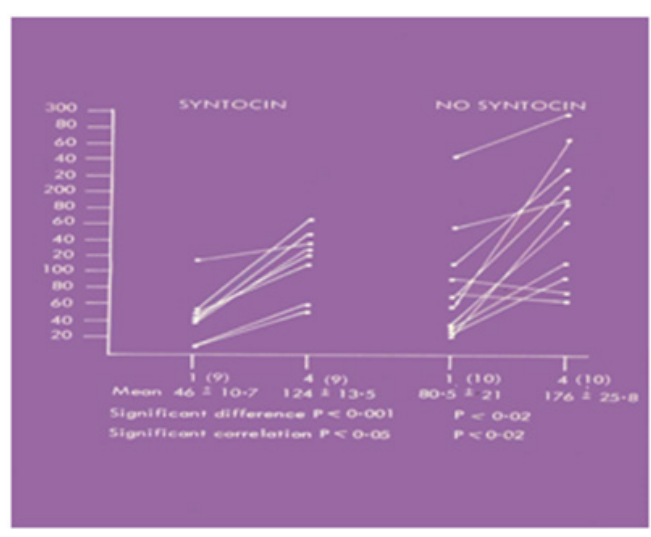

Figure I Fasting gastrin levels in 19 normal babies. Day I represents cord levels. The babies are separated into syntocin induced labours and normal labours. The syntocin babies demonstrate an individual correlation between Dal and Day 4.

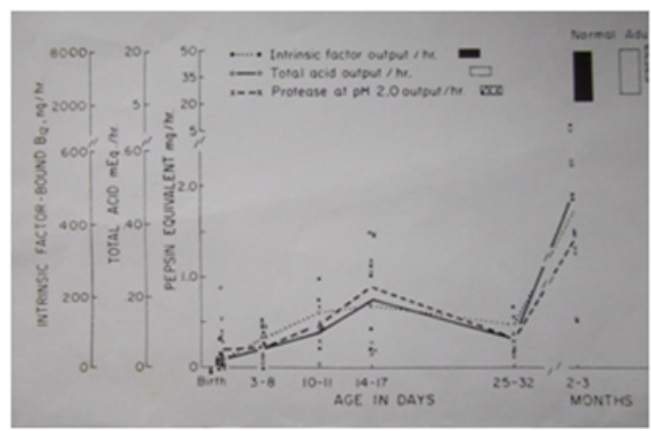

Figure 2 Total acid output can be shown to peak at around 17 days of age and thereafter fall before gradually resuming adult volume levels.

The known temporary peak acidity demonstrated by normal babies at 3 weeks of age is normally of no concern. However when coupled with an inherited hyperacidity, dangerously high acids will develop 
which will trigger a self-perpetuating process of acid-induced work hypertrophy of the sphincter. ${ }^{1}$ During the last 2-3 decades a significant and uniform decline in the incidence of PS has been reported across the Western world. ${ }^{7-9}$ Declines in incidence of $-38 \%$ have been reported from a live birth figure of $6,358,962 .{ }^{6}$ No similar decline has been reported from the undeveloped world. Between 1999 and 2004 a 7.5 fold rise in PPI prescriptions for babies under 12 months of age has occurred from a data base of 1,308,126 infants. ${ }^{10}$ Between 20042008 PPI prescriptions for newborns less than 1 month of age rose more than 2 fold from $0.07 \%$ to $0.17 \%$ - from a data base of 5000 which represents on average $0.13 \%$ of babies born. ${ }^{11}$ This incidence equates to the lower range of the incidence of PS. Most prescriptions were for an alleged but usually not confirmed diagnosis of GERD. There is no evidence that PPI drugs reduce reflux in the newborn. ${ }^{12}$ In 2011 the FDA legalized intra-venous esomeprazole for new-borns This is likely to further increase PPI usage. The duration of therapy is not defined. ${ }^{11,12}$ Vomiting or regurgitating infants will be unlikely to have escaped this treatment. Babies with PS also suffer from GERD with occasionally produces erosive acid induced reflux oesophagitis. It is of interest to also observe that in only $10 \%$ of pediatric patients is the diagnosis of GORD confirmed. ${ }^{11}$ What are we to make of this juxtaposition of decline in the incidence of PS and the rise in PPI prescriptions in the neonatal period? Could it be related? Could some of these alleged infantile GORD babies actually be babies with early PS? The closeness of the incidence of newborn PPI prescriptions to the incidence of PS means that inadvertent treatment of PS by PPI drugs is extremely likely. This must be particularly true if one considers only vomiting or regurgitating babies. The Hyperacidity theory clearly allows even temporary abolition of acidity at the right time to lead to a long term cure of PS. Un published data from South Africa report that when intra-venous cimetidine is given to babies with established PS whose sphincter diameter is no more than $3 \mathrm{~mm}$. 16 out of 17 babies are completely cured. ${ }^{12}$

\section{Conclusion}

a. The decline in incidence of PS in the Western world in recent decades is associated with an increase in PPI treatment of neonates during their first few weeks of age.

b. The decline is exceptional, uniform and hitherto unexplained. Demographic data suggest that the back to sleep regime primarily introduced to reduce the incidence of SIDS but subsequently advanced of a way of reducing PS, is not a major contributing factor. c. Reduction of acid secretion by PPI drugs at this critical age is advanced as a credible explanation for the decline in the incidence of PS in the Western World.

\section{Acknowledgments}

None.

\section{Conflicts of interest}

Author declares there are no conflicts of interest.

\section{Funding}

None.

\section{References}

1. D Lafollie, Turial S, Heckmann M, et al. Why pyloric stenosis of Infancy occurs-Simple echoes from the past. J Pediatr Neonatal Care. 2016;5(6): 00205.

2. Dodge JA, Karim AA. Induction of pyloric hypertrophy by pentagastrin. Gut. 1976;17(4):280-284.

3. Rogers IM, Davidson DC, Lawrence J ,et al. Neonatal secretion of gastrin and glucagon. Arch Dis Child. 1974;49(10):796-801.

4. Rodgers BM, Dix PM, Talbert JL. Fasting and post-prandial serum gastrin in normal human neonates. J Ped Surg. 1978;13(1):13-16.

5. Euler AR, Byrne WJ, Meisse PJ, et al. Basal and pentagastrin stimulated acid secretion in new born human infants Ped Res Jan. 1979;13(1):36-37.

6. Decline in IHPS in Germany in 2000-2008. Pediatrics . 2012;129(4): E901-906.

7. Hedback G, Abrahamson K, Husberg B, et al. The epidemiology of IHPS in Sweden 1987-96 Arch Dis Child . 2001;85(5): 379-381.

8. SommerfieldT,Chalmers J, Young son G, etal. The changing epidemiology of IHPS in Scotland. Arch Dis Child . 2008;93(12):1007-1011.

9. Ward RM, Kearns GL. Propton pump inhibitors in pediatrics. Paed Drugs. 2013;15(2):119-131.

10. Illveca M, Alemayehu B, Yang Huyung. Proton Pump inhibitor prescribing patterns in new borns and infants. J Pediatr Pharmacol Ther . 2014;19(4):283-287.

11. Omari T, Lundberg P, Sandstrom M. Pharmodynamics and systemic exposure of esomeprazole in preterm infants and term neonates with GERD J Pediatr. 2009; 155(2):222-228.

12. Banieghbal B. Personal communication. 2013. 